\title{
Electric-Field-Induced Magnetization Reversal in a Ferromagnet-Multiferroic Heterostructure
}

\author{
J. T. Heron, ${ }^{1}$ M. Trassin,,${ }^{2, *}$ K. Ashraf, ${ }^{3}$ M. Gajek, ${ }^{2}$ Q. He, ${ }^{2}$ S. Y. Yang, ${ }^{2}$ D. E. Nikonov, ${ }^{4}$ Y-H. Chu,,${ }^{2,5}$ \\ S. Salahuddin, ${ }^{3}$ and R. Ramesh ${ }^{1,2,6}$ \\ ${ }^{1}$ Department of Materials Science and Engineering, University of California, Berkeley, California 94720, USA \\ ${ }^{2}$ Department of Physics, University of California, Berkeley, California 94720, USA \\ ${ }^{3}$ Department of Electrical Engineering and Computer Science, University of California, Berkeley, California 94720, USA \\ ${ }^{4}$ Components Research, Intel Corporation, Santa Clara, California 95052, USA \\ ${ }^{5}$ Department of Materials Science and Engineering, National Chiao Tung University, Hsinchu 30010, Taiwan, Republic of China \\ ${ }^{6}$ Materials Science Division, Lawrence Berkeley National Laboratory, Berkeley, California 94720, USA
}

(Received 7 July 2011; published 14 November 2011)

\begin{abstract}
A reversal of magnetization requiring only the application of an electric field can lead to low-power spintronic devices by eliminating conventional magnetic switching methods. Here we show a nonvolatile, room temperature magnetization reversal determined by an electric field in a ferromagnet-multiferroic system. The effect is reversible and mediated by an interfacial magnetic coupling dictated by the multiferroic. Such electric-field control of a magnetoelectric device demonstrates an avenue for nextgeneration, low-energy consumption spintronics.
\end{abstract}

DOI: 10.1103/PhysRevLett.107.217202

PACS numbers: 75.85.+t, 75.60.Jk, 75.76.+j, 77.80.Dj

In the push for low-energy consumption memory and logic devices, the field of spintronics has focused on establishing control of magnetization without the need of a magnetic field. Because of carrier-mediated ferromagnetism, a current or electric field applied to a magnetic semiconductor has led to the control of the magnetization or a change of the magnetic anisotropy of this system [1-3]. Piezoelectric-ferromagnet heterostructures have also been used to demonstrate an electrical control of the magnetic anisotropy via strain transfer to the ferromagnetic layer $[4,5]$. A magnetization reversal requires injection or accumulation of spin-polarized carriers to transfer angular momentum to the macroscopic spin state [6-8] or to establish a large chemical potential gradient through nonlocal diffusion [9]. While these methods are effective, the large current densities required lead to a significant energy loss from heating. Promising alternatives and a rich field of physics reside in the use of magnetoelectric multiferroics $[10,11]$. An electric field can be applied to a multiferroic to alter its magnetic order [12-14]. Multiferroics that support both ferroelectric and magnetic orders are typically insulators with an antiferromagnetic spin arrangement $[15,16]$. Hence, to achieve electric-field control of ferromagnetism, multiferroics have been used in the form of ferromagnet-multiferroic heterostructures. The electricfield control of exchange bias has been successfully demonstrated in heterostructures containing $\mathrm{LuMnO}_{3}$ [17], $\mathrm{YMnO}_{3}$ [18], $\mathrm{Cr}_{2} \mathrm{O}_{3}$ [19,20], and $\mathrm{La}_{0.7} \mathrm{Sr}_{0.3} \mathrm{MnO}_{3} /$ $\mathrm{BiFeO}_{3}$ [21]. In the above works, the need for low temperatures or an applied magnetic field (or the combination) to control the exchange bias and magnetization reversal make these systems unfit for practical applications. The purely electric-field control of a magnetization has been achieved at room temperature using $\mathrm{BiFeO}_{3}$ crystals and films [22,23]; however, these studies were unable to achieve an $180^{\circ}$ reversal of a magnetization. In this study, we probed the anisotropic magnetoresistance (AMR) of a ferromagnetic $\mathrm{Co}_{0.90} \mathrm{Fe}_{0.10}(\mathrm{CoFe})$ layer in contact with multiferroic $\mathrm{BiFeO}_{3}$ (BFO). We show the reversible and deterministic reversal of the ferromagnet's magnetization at room temperature, in the absence of a magnetic field, through the application of an electric field to the $\mathrm{CoFe} / \mathrm{BFO}$ heterostructure. A combination of soft $\mathrm{x}$-ray and piezoresponse force microscopy (PFM) studies reveal that $\mathrm{CoFe}$ couples to the canted moment in the BFO layer and that the reversal of the net in-plane projection of BFO's polarization induces the magnetoelectric switching necessary for electric-field reversal of the $\mathrm{CoFe}$ magnetization.

BFO (001) films 70-100 nm thick were grown on (110)-oriented $\mathrm{DyScO}_{3}$ (DSO) substrates [24]. The ferroelectric domain architecture was probed using PFM. The anisotropic strain imposed by DSO substrates [25] resulted in the formation of a simple, quasiperiodic, ferroelectric domain architecture (domain width of $\sim 150 \mathrm{~nm}$ ) where, in this as-grown state, only two ferroelectric polarization variants are separated by $71^{\circ}$. The sum of these two polarization variants is a well-defined in-plane projection of the net polarization $\left(P_{\text {net IP }}\right)$ which points along the [1-10] direction of the DSO substrate.

To probe interfacial coupling of a ferromagnetmultiferroic heterostructure, $\mathrm{Pt}(2.5 \mathrm{~nm}) / \mathrm{CoFe}(2.5 \mathrm{~nm})$ bilayers were grown on BFO films in a 200 Oe magnetic field. Figure 1(a) presents in-plane hysteresis loops, obtained by vibrating sample magnetometry, taken from samples where the growth field was applied perpendicular (red circles) and parallel (black circles) to $P_{\text {net IP. The }}$ data in Fig. 1(a) clearly illustrate that, irrespective of the orientation of the growth field, the anisotropy is always 


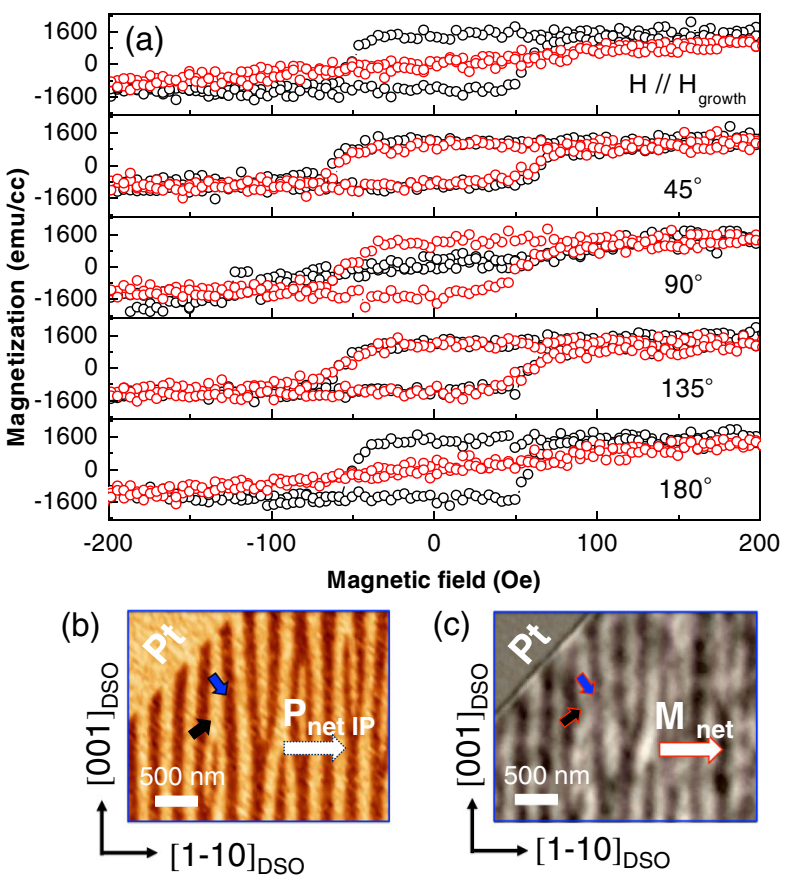

FIG. 1 (color online). (a) In-plane $M(H)$ curves measured every $45^{\circ}$ at room temperature from $\mathrm{CoFe} / \mathrm{BFO}$ heterostructures. The CoFe growth field was applied along (black open circles) or perpendicular [gray (red) open circles] to the net inplane polarization direction $\left(P_{\text {net IP }}\right)$. (b) In-plane PFM image of BFO. (c) XMCD-PEEM image of the CoFe/BFO heterostructure. The gray (blue) and black arrows in (b) and (c) correspond to the in-plane projections of the polarizations in each of the ferroelectric domains of BFO and to the magnetic moments in the $\mathrm{CoFe}$ layer, respectively.

uniaxial and along the axis corresponding to the direction

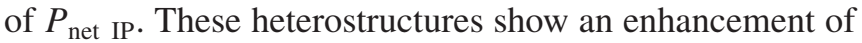
the coercive field, compared to CoFe grown on DSO substrate, and negligible exchange bias anisotropy. The insertion of a fully strained, epitaxial $\mathrm{SrTiO}_{3}(2 \mathrm{~nm})$ or $\mathrm{SrRuO}_{3}$ $(2 \mathrm{~nm})$ spacer between $\mathrm{BFO}$ and $\mathrm{CoFe}$ resets the easy axis of the CoFe films to the direction of the applied growth field, ruling out stress induced magnetic annealing as the cause of this preferential anisotropy axis.

To investigate the microscopic origins of this coupling, the magnetic state of the CoFe layer was imaged using $\mathrm{x}$-ray magnetic circular dichroism (XMCD) photoemission electron microscopy (PEEM) at the Co $L_{3}$ edge. The BFO ferroelectric domain structure was imaged by PFM following the removal of CoFe by ion milling [see Fig. 1(b)]. The XMCD Co images were obtained from the ratio between PEEM images taken with right- and left-polarized $\mathrm{x}$ rays, resulting in a $2 \mathrm{D}$ map of projected magnetization of the ferromagnetic Co domains along the direction of incident $\mathrm{x}$ rays. Figure 1(c) reveals two distinct contrast levels, corresponding to two in-plane ferromagnetic domains. The CoFe adapts a domain ordering that matches BFO's domain pattern. XMCD-PEEM images were taken at different orientations of the sample relative to incoming $\mathrm{x}$ rays to distinguish the orientation of magnetization within each domain. The directions of the underlying ferroelectric domain polarizations and $\mathrm{CoFe}$ domain magnetizations are given in Figs. 1(b) and 1(c), respectively. The magnetization within each ferromagnetic domain is found to be collinear with the direction of the in-plane projection of the polarization of the corresponding ferroelectric domain in BFO. Considering a single BFO unit cell with a polarization $(P)$ pointing along a $\langle 111\rangle$ direction, the canted moment $\left(M_{c}\right)$ and the antiferromagnetic axis $(L)$ lie perpendicular to each other in the (111) plane [26]. Since this coupling has been determined to be magnetic in nature, the collinear correlation observed in Figs. 1(b) and 1(c) suggests that BFO's canted moment is pointing along the $\langle 11-2\rangle$, which projects parallel to the polarization on the (001) surface and leads to a parallel arrangement of both $P_{\text {net IP }}$ and the net CoFe magnetization as shown in Figs. 1(b) and 1(c).

The interfacial coupling between the ferromagnet and $\mathrm{BFO}$, combined with the intrinsic magnetoelectric correlations in the multiferroic film [27], implies that the magnetization of the ferromagnet can be controlled within the realms of ferroelectric switching allowed in BFO. Since the polarization of a single ferroelectric domain in the BFO system must be along one of the eight possible $\langle 111\rangle$ directions, the polarization can only be switched by $71^{\circ}$, $109^{\circ}$, or $180^{\circ}$ [28]. Considering the projection onto the BFO (001) surface, the in-plane $71^{\circ}$ switching event is accompanied by a rotation of the magnetic (111) plane that corresponds to a $90^{\circ}$ in-plane rotation of the projected antiferromagnetic axis. This change should induce an associated rotation of the in-plane projection of the canted moment. Using a striped two-variant BFO film, the combination of each of the polarization variants rotating by $71^{\circ}$ (i.e., $90^{\circ}$ in-plane-one rotates clockwise while the other rotates counterclockwise), can lead to a unique path of a reversal of $P_{\text {net IP. }}$ Because of the one-to-one magnetic interface coupling in our heterostructures, a reversal of $P_{\text {net IP }}$ in this way suggests that a reversal of the in-plane projection of BFO's magnetic order and the magnetization of the exchange coupled $\mathrm{CoFe}$ layer is possible.

The architecture shown in Fig. 2(a) was designed for angle-dependent AMR measurements to probe the magnetic state of the CoFe dots as a function of applied electric field to the BFO layer. Two outer poling electrodes on the surface of BFO are dedicated to the in-plane ferroelectric switching of BFO. Two leads make contact to a $2 \times 4 \mu \mathrm{m}^{2}$ $\mathrm{CoFe}$ dot positioned at the center of the gap, between the poling electrodes.

To determine the viability of a $P_{\text {net IP }}$ reversal in this AMR architecture, the ferromagnetic dot was removed by $\mathrm{Ar}$ ion milling to reveal the ferroelectric state of the underlying BFO film after electrical poling. Figures 2(a) and 2(b) show the in-plane PFM images of an as-grown AMR structure before and after the etching of the 


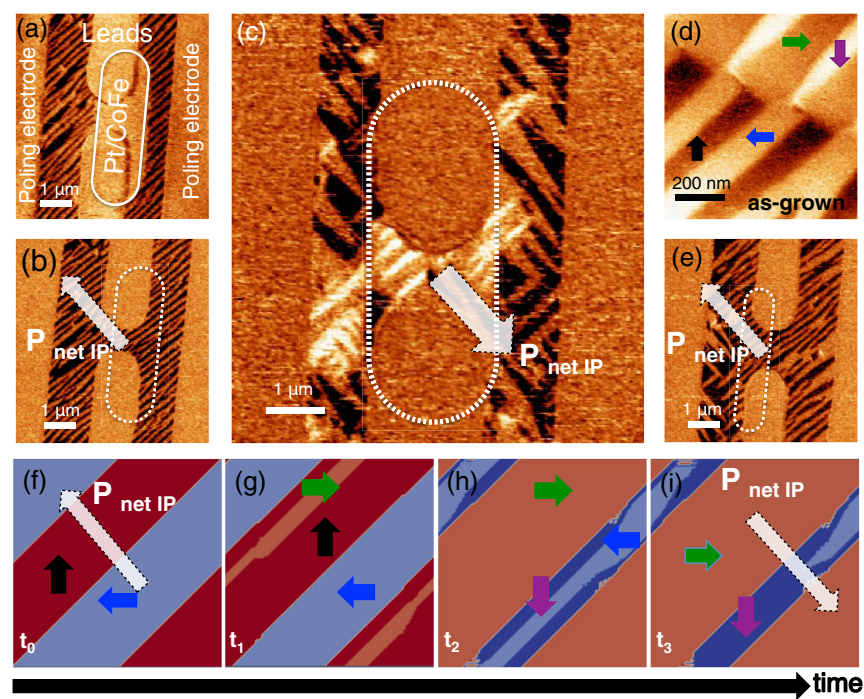

FIG. 2 (color online). (a),(b) In-plane PFM images before (a) and after (b) removal of the CoFe dot from an as-grown AMR structure. The white arrow gives the direction of $P_{\text {net IP }}$ underneath the CoFe dot. (c) After the application of a $130 \mathrm{kV} \cdot \mathrm{cm}^{-1}$ pulse. (d) IP-PFM image where both as-grown and reversed states of $P_{\text {net IP }}$ coexist, the rotation of each single ferroelectric domain is represented by the arrows. (e) Another structure which has been switched by a $130 \mathrm{kV} \cdot \mathrm{cm}^{-1}$ and by a $-130 \mathrm{kV}$. $\mathrm{cm}^{-1}$ pulse. (f)-(h) Phase field simulation as a function of increasing time of the ferroelectric switching.

ferromagnetic dot. The dashed open white arrows give the directions of $P_{\text {net IP }}$ under the CoFe dot. Continuity of the $71^{\circ}$ ferroelectric domain stripes after removing the $\mathrm{CoFe}$ shows that controlled etching allows a clear visualization of the domain configuration underneath the ferromagnet without altering the ferroelectric domain state. After a $130 \mathrm{kV} \cdot \mathrm{cm}^{-1}$ pulse, the in-plane contrast change observed where the CoFe dot once sat [Fig. 2(c)] reveals that $P_{\text {net IP }}$ has rotated by $180^{\circ}$ with respect to the as-grown state. No change in the out-of-plane polarization direction was observed. Figure 2(d) presents the ferroelectric architecture of the BFO film where both as-grown and $180^{\circ}$ switched states coexist. The continuity of ferroelectric domain walls evinces that the $180^{\circ}$ switch of $P_{\text {net IP }}$ consists of two $71^{\circ}$ rotations of the single ferroelectric domains and confirms the $180^{\circ}$ switching event of $P_{\text {net IP. }}$. Reversibility of the switching is shown in Fig. 2(e) where two successive switching pulses $\left(130 \mathrm{kV} \cdot \mathrm{cm}^{-1}\right.$ followed by $-130 \mathrm{kV} \cdot \mathrm{cm}^{-1}$ ) were applied to a third structure.

Electric-field-driven phase field simulations reveal the origin of the observed $P_{\text {net IP }}$ reversal underneath the $\mathrm{CoFe}$ dot. Prior studies have suggested that the $71^{\circ}$ and $109^{\circ}$ switching events in BFO are characterized by two different coercive fields with $E_{c, 71^{\circ}}<E_{c, 109^{\circ}}$ [29]. Considering the as-grown $71^{\circ}$ striped BFO configuration represented in Fig. 2(f), the high saturation polarization $\left(\sim 90 \mu \mathrm{C} \cdot \mathrm{cm}^{-2}\right)$ of BFO causes all of the domains to arrange in-plane in a head-to-tail configuration so that the dipole-dipole energy is minimized. For an applied electric field of strength $E_{c, 109^{\circ}}>E_{\text {applied }}>E_{c, 71^{\circ}}$, the time evolution of the system demonstrates that the ferroelectric domains with an in-plane polarization oriented perpendicular to the applied electric field align first with the external field [Fig. 2(g)]. This corresponds to a $71^{\circ}$ switching event which nucleates at the domain wall, generating an energetically unfavorable head-to-head configuration. Adjacent domains originally oriented antiparallel to the electric field switch in-plane by $90^{\circ}$ (corresponding to a second $71^{\circ}$ switching event) under this dipole-dipole field to recover the preferred head-totail configuration of the polarizations [Fig. 2(h)]. $P_{\text {net IP }}$ changes by $180^{\circ}$, permitting the in-plane projection of the antiferromagnetic order to reverse without domain wall reorientation [Fig. 2(i)].

The consequential effect of the $180^{\circ}$ rotation of $P_{\text {net IP }}$ on the canted moment in the BFO layer (and thus on the CoFe magnetic moment) was probed using AMR measurements. In a conventional ferromagnet, the AMR follows a $R(\theta)=R_{0}+\left(R_{\|}-R_{0}\right) \cos ^{2}(\theta)$ dependence [30], where $\theta$ is the angle between magnetization and the current, while $R_{0}$ and $R_{\|}$are the resistances when $\theta=90^{\circ}$ and $0^{\circ}$. High magnetic field (2000 Oe) resistance data taken from the as-grown state shows a $\cos ^{2}\left(\theta_{a}\right)$ dependence [black curve in Fig. 3(a)], where $\theta_{a}$ is the angle of the applied magnetic field. At this field, the Zeeman energy dominates the other anisotropies and $\mathrm{CoFe}$ magnetization follows the applied magnetic field (i.e., $\theta \sim \theta_{a}$ ). At a low magnetic field of $20 \mathrm{Oe}$, the resistance follows a $\cos \left(\theta_{a}\right)$-like dependence [red circles in second panel from top in Fig. 3(a)]. At this low field the magnetization simply wiggles about the dominant anisotropy axis that is $\sim 45^{\circ}$ away from the current direction [31]. The deduced orientation of the magnet with respect to $P_{\text {net IP }}$ is in agreement with the interfacial coupling evidenced previously and schematically represented in Fig. 3(b). The large coercivity of the CoFe hysteresis, due to the exchange coupling with BFO, prevents the $\mathrm{CoFe}$ magnetization from switching under the low magnetic field of 20 Oe. The AMR following a $130 \mathrm{kV} \cdot \mathrm{cm}^{-1}$ (under zero magnetic field) electric-field pulse is presented as the blue circles in Fig. 3(a). The AMR retains the $\cos \left(\theta_{a}\right)$-type behavior; there is a $180^{\circ}$ phase shift when compared to the as-grown state. This $180^{\circ}$ phase change is the result of a change in the sign of the magnetic torque experienced by the magnetization, due to a magnetization reversal. The corresponding schematization is represented in Fig. 3(c). Furthermore, below $\sim 100 \mathrm{kV} \cdot \mathrm{cm}^{-1}$ no change in the AMR response was observed. The data in Fig. 3(a) are representative of a large sample set ( $\sim 20$ samples), where such switching has been observed. Additionally, the state after switching is observed to be stable for over $15 \mathrm{~h}$, demonstrating its nonvolatile nature. Finally, the BFO was poled with a $-130 \mathrm{kV} \cdot \mathrm{cm}^{-1}$ pulse and AMR data were plotted in 


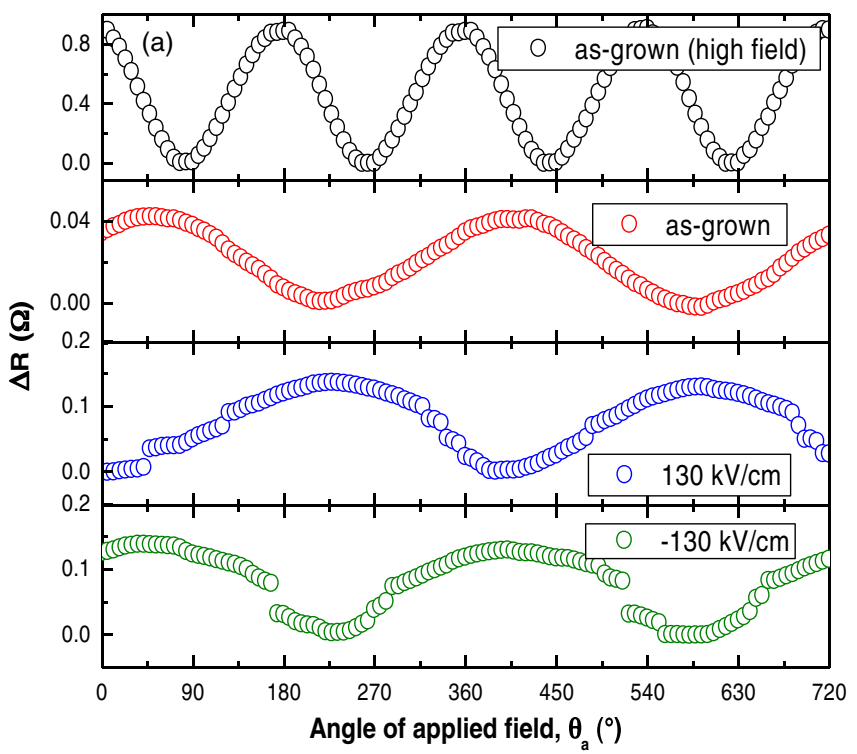

(b)

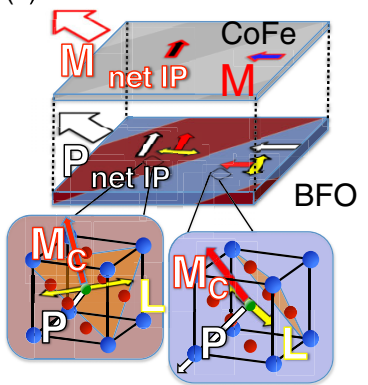

(c)

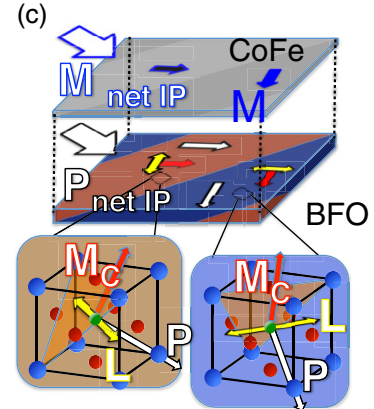

FIG. 3 (color online). (a) Open black circles show the high field (2000 Oe) AMR response (top panel). The low-field (20 Oe) AMR response for the as-grown state is plotted with the open red circles (second panel from top). The open blue circles show the low-field AMR after pulsing an electric field of $130 \mathrm{kV} \cdot \mathrm{cm}^{-1}$ in zero magnetic field (second panel from bottom). Application of a $-130 \mathrm{kV} \cdot \mathrm{cm}^{-1}$ electric-field pulse results in the recovery of the phase of the as-grown low-field AMR response (open green circles, bottom panel). (b),(c) Representations of the one-to-one magnetic interface coupling in the $\mathrm{CoFe} / \mathrm{BFO}$ heterostructure in the (b) as-grown state and (c) after the first electric pulse.

green in the bottom panel of Fig. 3(a), which is in phase with the as-grown curve. This illustrates that the magnetization has again reversed by $180^{\circ}$, back into the as-grown direction. In comparison, no change in the low-field AMR curve after poling was observed for a heterostructure where a $2 \mathrm{~nm} \mathrm{SrTiO}_{3}$ spacer was inserted between $\mathrm{BFO}$ and $\mathrm{CoFe}$, indicating that magnetostriction is not the origin of this effect.

In summary, we have demonstrated the ability to reverse magnetization of a ferromagnet solely with an electric field by exploiting the magnetic coupling at the interface between a ferromagnetic layer and the magnetoelectric multiferroic $\mathrm{BiFeO}_{3}$ and the intrinsic magnetoelectric switching of $\mathrm{BiFeO}_{3}$. This heretofore unreported method of magnetization reversal is a critical advancement in the field of spintronics, providing a unique pathway to writing a magnetic state. An extension of this demonstration would be to couple a spin valve device to the $\mathrm{BiFeO}_{3}$ layer. Such a design would allow for the read and write operations to be performed on the same device using only small currents for reading and an electric field for writing.

We sincerely thank J. Ravichandran and O. Sapunkov for the thoughtful discussions and measurement assistance, respectively. K. A. and S.S. acknowledge support from Nanoelectronic Research Initiative (NRI) and the National Science Foundation (NSF). The authors acknowledge the support from the DOD-ARO MURI. J. T.H acknowledges that this research was made with Government support under and awarded by DOD, Air Force Office of Scientific Research, National Defense Science and Engineering Graduate (NDSEG) Fellowship, 32 CFR 168a.

*mtrassin@ berkeley.edu

[1] H. Ohno et al., Nature (London) 408, 944 (2000).

[2] D. Chiba et al., Nature (London) 455, 515 (2008).

[3] A. Chernyshov et al., Nature Phys. 5, 656 (2009).

[4] S. Sahoo et al., Phys. Rev. B 76, 092108 (2007).

[5] S. Geprägs, A. Brandlmaier, M. Opel, R. Gross, and S. T. B. Goennenwein, Appl. Phys. Lett. 96, 142509 (2010).

[6] E. B. Myers, D. C. Ralph, J.A. Katine, R. N. Louie, and R. A. Buhrman, Science 285, 867 (1999).

[7] D. C. Ralph and M. D. Stiles, J. Magn. Magn. Mater. 320, 1190 (2008).

[8] J. C. Slonczewski, J. Magn. Magn. Mater. 159, L1 (1996).

[9] T. Yang, T. Kimura, and Y. Otani, Nature Phys. 4, 851 (2008).

[10] N. A. Spaldin and M. Fiebig, Science 309, 391 (2005).

[11] W. Eerenstein, N.D. Mathur, and J.F. Scott, Nature (London) 442, 759 (2006).

[12] D. Lebeugle et al., Phys. Rev. Lett. 100, 227602 (2008).

[13] S. Lee, W. Ratcliff II, S-W. Cheong, and V. Kiryukhin, Appl. Phys. Lett. 92, 192906 (2008).

[14] T. Lottermoser et al., Nature (London) 430, 541 (2004).

[15] N. A. Hill, J. Phys. Chem. B 104, 6694 (2000).

[16] D. I. Khomskii, J. Magn. Magn. Mater. 306, 1 (2006).

[17] V. Skumryev et al., Phys. Rev. Lett. 106, 057206 (2011).

[18] V. Laukhin et al., Phys. Rev. Lett. 97, 227201 (2006).

[19] P. Borisov, A. Hochstrat, X. Chen, W. Kleemann, and C. Binek, Phys. Rev. Lett. 94, 117203 (2005).

[20] X. He et al., Nature Mater. 9, 579 (2010).

[21] S. M. Wu et al., Nature Mater. 9, 756 (2010).

[22] Y. H. Chu et al., Nature Mater. 7, 478 (2008).

[23] D. Lebeugle, A. Mougin, M. Viret, D. Colson, and L. Ranno, Phys. Rev. Lett. 103, 257601 (2009).

[24] Y. H. Chu et al., Nano Lett. 9, 1726 (2009). 
[25] Y. H. Chu et al., Adv. Mater. 18, 2307 (2006).

[26] C. Ederer and N.A. Spaldin, Phys. Rev. B 71, 060401 (2005).

[27] T. Zhao et al., Nature Mater. 5, 823 (2006).

[28] S. K. Streiffer et al., J. Appl. Phys. 83, 2742 (1998).
[29] S. H. Baek et al., Nature Mater. 9, 309 (2010).

[30] T. R. McGuire and R. I. Potter, IEEE Trans. Magn. 11, 1018 (1975).

[31] B. H. Miller and E. Dan Dahlberg, Appl. Phys. Lett. 69, 3932 (1996). 